Article

\title{
On Modeling the Bending Stiffness of Thin Semi-Circular Flexure Hinges for Precision Applications
}

\author{
Mario André Torres Melgarejo ${ }^{1}$, Maximilian Darnieder ${ }^{1, *(\mathbb{D},}$, Sebastian Lin ${ }^{2}{ }^{\mathbb{D}}$, \\ Lena Zentner ${ }^{2}$, , Thomas Fröhlich ${ }^{3}$ (D) and René Theska ${ }^{1}$ (D) \\ 1 Precision Engineering Group, Institute for Design and Precision Engineering, Department of Mechanical \\ Engineering, Technische Universität Ilmenau, 98693 Ilmenau, Germany; \\ mario-andre.torres-melgarejo@tu-ilmenau.de (M.A.T.M.); rene.theska@tu-ilmenau.de (R.T.) \\ 2 Compliant Systems Group, Department of Mechanical Engineering, Technische Universität Ilmenau, \\ 98693 Ilmenau, Germany; sebastian.linss@tu-ilmenau.de (S.L.); lena.zentner@tu-ilmenau.de (L.Z.) \\ 3 Process Measurement Technology Group, Institute of Process Measurement and Sensor Technology, \\ Department of Mechanical Engineering, Technische Universität Ilmenau, 98693 Ilmenau, Germany; \\ thomas.froehlich@tu-ilmenau.de \\ * Correspondence: maximilian.darnieder@tu-ilmenau.de; Tel.: +49-3677-69-3826
}

Received: 30 October 2018; Accepted: 4 December 2018; Published: 6 December 2018

\begin{abstract}
Compliant mechanisms based on flexure hinges are widely used in precision engineering applications. Among those are devices such as precision balances and mass comparators with achievable resolutions and uncertainties in the nano-newton range. The exact knowledge of the mechanical properties of notch hinges and their modeling is essential for the design and the goal-oriented adjustment of these devices. It is shown in this article that many analytical equations available in the literature for calculating the bending stiffness of thin semi-circular flexure hinges cause deviations of up to $12 \%$ compared to simulation results based on the three-dimensional finite element model for the considered parameter range. A close examination of the stress state within the loaded hinge reveals possible reasons for this deviation. The article explains this phenomenon in detail and shows the limitations of existing analytical models depending on specific geometric ratios. An accurate determination of the bending stiffness of semi-circular flexure hinges in a wide range of geometric parameters without the need for an elaborate finite element analysis is proposed in form of FEM-based correction factors for analytical equations referring to Euler-Bernoulli's beam theory.
\end{abstract}

Keywords: compliant mechanism; flexure hinge; bending stiffness; finite element method

\section{Introduction}

Due to their monolithic design, compliant mechanisms have significant advantages over rigid-body mechanisms for precision engineering applications and metrology [1-4] (e.g., Figure 1). Negligible friction, no stick-slip behavior, lack of clearance and backlash as well as smooth and highly repeatable motion are to be mentioned here. Their advantages further include compactness and lack of wear and maintenance. Since lubrication is not required, they are well suited for clean room and vacuum applications. Disadvantages of these mechanisms comprise limited motion, due to the tolerable stress (or strain), as well as inherent non-pure rotation of the flexure hinges.

Flexure hinges are materially coherent connections between two bodies or body segments with at least one relative motion due to mostly elastic deformation [5]. Typical notch flexure hinges, as in Figure 2, allow a relative rotation through bending of its neck-like region with concentrated compliance. The performance of compliant mechanisms in force measurement applications depends largely on 
the compliance or low stiffness of their joints. In those instruments, the rotation of the flexure hinges is limited by mechanical stops and a position control during operation, resulting in deflection ranges $<<1^{\circ}$. The bending stiffness defines the sensitivity of the force measurement. To enhance the sensitivity, the minimum notch height $h$ of the flexure hinges is reduced to a few hundredths of a millimeter [6,7] (see Figure 1). This leads to difficulties in the accurate determination of the bending stiffness of very thin flexure hinges, since the assumptions of commonly used analytical modeling approaches no longer apply. With small notch heights, the mechanical properties become more sensitive to small imperfections, e.g., manufacturing and material related. To avoid parasitic motions, the lateral stiffnesses are usually increased by setting the width $b$ very large in comparison to the minimum notch height $h$.

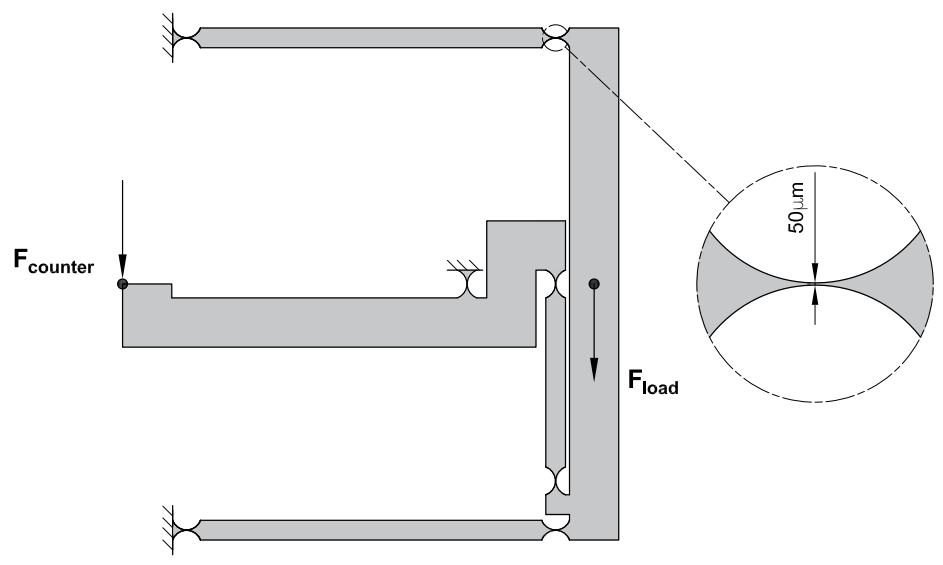

Figure 1. Simplified representation of a compliant mechanism used in a weighing cell in [1].

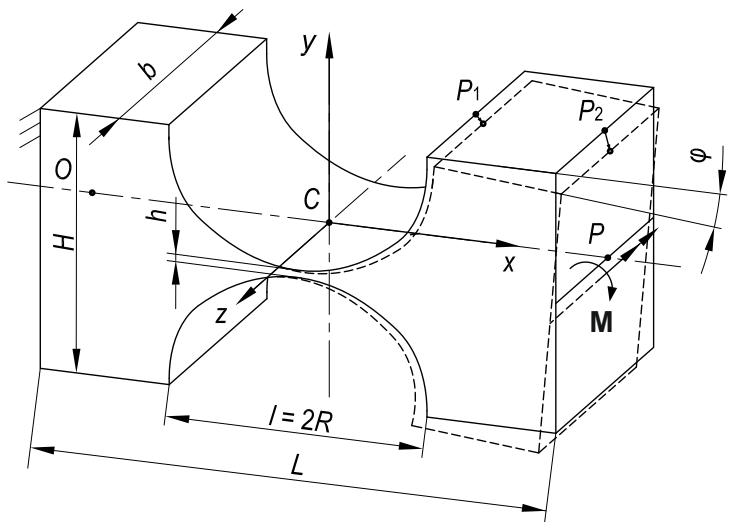

Figure 2. Model and geometric parameters of a semi-circular flexure hinge.

The article highlights the existing deviation of the bending stiffness calculated by known analytical equations and finite element models in comparison. The remaining sections are structured as follows. Section 2 discusses the approaches for the modeling of flexure hinges. Section 3 deals with the finite element (FE) modeling of thin flexure hinges. The deviation between results of analytical and FE models is explained in Section 4. An approach for an accurate determination of the bending stiffness based on correction factors and its use in application examples are presented in Sections 5 and 6, respectively. Finally, conclusions are drawn in Section 7.

\section{Approaches for Modeling the Bending Stiffness of Flexure Hinges}

The literature provides numerous geometries for flexure hinges [8,9] with the semi-circular notch contour being the most prevailed (see Figure 2). Most applications are based on single-axis notch flexure hinges to realize planar or spatial motions. Common approaches for the quasi-static modeling 
of these flexure hinges consider the notch contour as a beam with variable rectangular cross-section, i.e., variable height $h(x)$, with one end fixed and the load applied on the opposing end. Due to the resulting deformation, the cross-section at the loaded end is displaced and rotated. The relative motion between both ends (points $\mathrm{O}$ and $\mathrm{P}$ in Figure 2) can be formulated as:

$$
\mathbf{F}=\mathbf{K} \mathbf{u}
$$

Here $\mathbf{F}$ denotes the load vector, $\mathbf{u}$ the displacement vector and $\mathbf{K}$ the stiffness matrix. In general terms, $\mathbf{K}$ is a $6 \times 6$ matrix whose components describe the relationship between the components of the load and displacement vectors. To simplify the synthesis of compliant mechanisms, a flexure hinge is often modeled as a revolute joint with a torsional spring. The position of the substitute joint and the stiffness of the spring depend on the geometry and loading conditions of the flexure hinge [10]. For hinges with concentrated compliance, common in high-precision systems [1,2,4], this location can be assumed at their geometrical center (point $C$ in Figure 2) [11]. This way, the stiffness of the equivalent spring no longer depends on the type of load [10]. In analogy to the rotational stiffness of the spring, the bending stiffness $k_{\varphi}$ of these hinges can be approximated by

$$
M=k_{\varphi} \varphi,
$$

where $M$ is the applied bending moment and $\varphi$ the resulting rotation angle, both about the $z$-axis. Based on this characterization, many authors have derived analytical and approximate equations for calculating the bending stiffness using different theories. A survey of existing modeling approaches and common model assumptions $[8,10]$ in the literature is presented in Table 1.

Table 1. Approaches and assumptions for quasi-static modeling of the bending stiffness of notch flexure

\begin{tabular}{|c|c|c|c|c|c|}
\hline Approach & Equation/Model by & Contour & 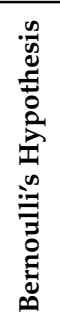 & 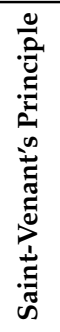 & 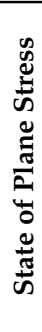 \\
\hline Euler-Bernoulli's beam theory & Paros and Weisbord [12] & $\mathrm{C}$ & $x$ & $x$ & X \\
\hline Castigliano's second theorem & Lobontiu [9] & $C, C F, E, P$ & $x$ & $x$ & X \\
\hline Principle of virtual work & Li et al. [13] & PF & $x$ & $x$ & $x$ \\
\hline Finite Beam-based Matrix Modelling & Zhu et al. [14] & $C, E, P F$ & $x$ & $x$ & $x$ \\
\hline Timoshenko's beam theory & Dirksen and Lammering [15] & C, P & & $x$ & X \\
\hline Continuum mechanics & Tseytlin [16] & $C, E, P$ & & & \\
\hline Nonlinear theory of large deflections & Linß et al. [17] & C, CF, E, PL & $x$ & $x$ & $x$ \\
\hline \multirow[t]{2}{*}{ of rod-like structures [10] } & Henning et al. [18] & C, CF, E, PL & $x$ & $x$ & X \\
\hline & Smith et al. [2] & $\mathrm{C}, \mathrm{E}$ & & & $x$ \\
\hline \multirow[t]{2}{*}{ Finite element method } & Koster et al. [19] & $\mathrm{C}$ & & & X \\
\hline & Schotborgh et al. [20] & $\mathrm{C}, \mathrm{CF}$ & & & $x$ \\
\hline
\end{tabular}
hinges (C: Circular; CF: Corner-filleted; E: Elliptical; P: Parabolic; PF: Power function; PL: Polynomial).

The assumptions in Table 1 serve as the basis for deriving the well-known Euler-Bernoulli's bending differential equation. In fact, the analytical equations by Paros and Weisbord [12], Lobontiu [9] and Li et al. [13] are exact solutions of the differential equation for various notch contours using different methods. Zhu et al. [14] proposes a numerical approach to determine the overall compliance by summating the compliances of a finite number of short Euler-Bernoulli beams. Other approaches have been used to obtain more accurate and tractable equations, especially the FE method $[2,19,20]$. Dirksen and Lammering [15] take the often-neglected shear deformation into account to derivate equations for rectangular flexure hinges. The equations by Tseytlin [16] are approximations for 
different geometry ranges from the plane equations of continuum mechanics. In contrast to the previous approaches, the nonlinear theory in [10] considers large deformations of rod-like structures and can be only solved numerically, as implemented in the design tool by Henning et al. [18]. The contour-dependent design equations by Linß et al. [17] are approximated from the results obtained using the model equations in [10]. These simplifications produce important deviations in the determination of the bending stiffness of flexure hinges with general dimensions [21,22].

Other effects, often neglected in analytical approaches, are to be expected in flexure hinges. Among them are stress concentration [15] and anticlastic bending [23]. On the one hand, a changing cross-section generates peaks of the bending stress in the thinnest region. On the other hand, the anticlastic shape due to the Poisson's effect in plates under bending is restrained producing a local state of plane strain at the center region. It is important to remark the effects unrelated to modeling that can alter the bending stiffness, e.g., axial loads [24] or manufacturing imperfections [25]. Modeling deviations are accepted by some authors since they lie near those caused by manufacturing errors [15]. However, this cannot be consistently assumed due to the vast number of parameters involved. A more extensive study on this matter is yet to be done, especially for very thin flexure hinges, which are typical in high-precision measurement instruments.

Most of the assumptions in Table 1 lead to small errors in precision engineering applications. However, the assumption of a plane stress state is problematic due to the large aspect ratios of thin flexure hinges. The validity of this assumption needs to be verified with the help of FE models. The FE model itself must be carefully prepared to reduce the influence of other effects and to achieve a meaningful interpretation of later results.

\section{Finite Element Modeling of Thin Flexure Hinges}

The investigation on the deviation between different models requires the selection of a suitable notch contour based on its significance in the field of application. Semi-circular contours are widely used in precision engineering, especially in weighing technology, due to their manufacturability with conventional processes and a high degree of motion accuracy for thin geometries $[3,8]$. Its widespread use has led to the development of numerous modeling equations $[2,9,12,16,17,20]$. The present investigation focusses on semi-circular flexure hinges which can be considered thin, as the one shown in Figure 2.

There is no consistent definition of "thin" flexure hinges in the literature. In [16], thin flexure hinges are defined as those whose deformation occurs mostly within the boundaries of the notch contour, which is a very general statement. Semi-circular contours are further classified in [16] according to the ratio of minimum notch height $h$ to radius $R$ as: thin $(h / R \leq 0.07)$, intermediate $(0.07<h / R \leq 0.2)$ and thick $(0.2<h / R \leq 1.0)$. Thus, thin flexure hinges are mainly those with very small notch heights $h$. The current limit in terms of manufacturing technology lies around $50 \mu \mathrm{m}$ [7]. For the thinnest hinges, the typical ratio of width $b$ to minimum notch height $h$ is about 200 [26], with ratios up to 375 still being achievable [6]. The geometrical dimensions of the FE model of the flexure hinge were chosen according to typical values in precision measurement applications (see Table 2). The material for this investigation is a high-strength aluminum alloy with an assumed linear elastic behavior [27].

The simple geometry of a notch flexure hinge allows the use of a mapped mesh with hexahedral elements. However, the large aspect ratios of very thin hinges require special consideration. Here, an optimum between element quality and number of elements must be found. This can be achieved by using an appropriate meshing strategy. The proposed strategy shown in Figure 3a and considers the following settings:

- The domain is divided into three parts: a highly refined central zone (A), a transition zone (B) and a roughly meshed outer zone (C).

- The boundary between the central zone (A) and the transition zone (B) is controlled so that the local normal stress in the $x$-direction is $10 \%$ of its maximum value. 
Table 2. Parameter values used for the flexure hinge model.

\begin{tabular}{lcc}
\hline Parameter & Symbol & Value \\
\hline Total length & $L$ & $15 \mathrm{~mm}$ \\
Total height & $H$ & $9 \mathrm{~mm}$ \\
Radius & $R$ & $3 \mathrm{~mm}$ \\
Minimum notch height & $h$ & $50 \mu \mathrm{m}$ \\
Width & $b$ & $10 \mathrm{~mm}$ \\
Young's modulus & $E$ & $71 \mathrm{GPa}$ \\
Poisson's coefficient & $v$ & 0.33 \\
Density & $\rho$ & $2.8 \mathrm{~g} / \mathrm{cm}^{3}$ \\
\hline
\end{tabular}

- The transition zone (B) is limited by the transition of the notch contour to the adjacent links.

- In the central zone (A), the element size in the $z$-direction depends on the minimum element size in the $x-y$ plane and a maximum aspect ratio of the hexahedral elements.

- To reduce the distortion of the elements when adapting to the semi-circular contour, the zones are delimited by cylindrical surfaces.

- The non-matching meshes are connected at their interface by suitable contact elements.

- Elements with quadratic approximation functions are used to avoid shear locking.

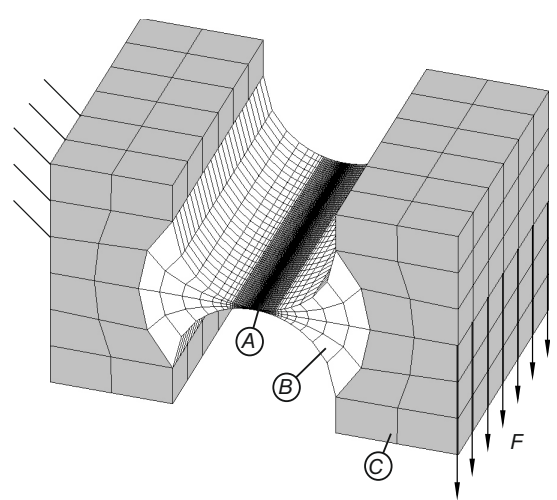

(a)

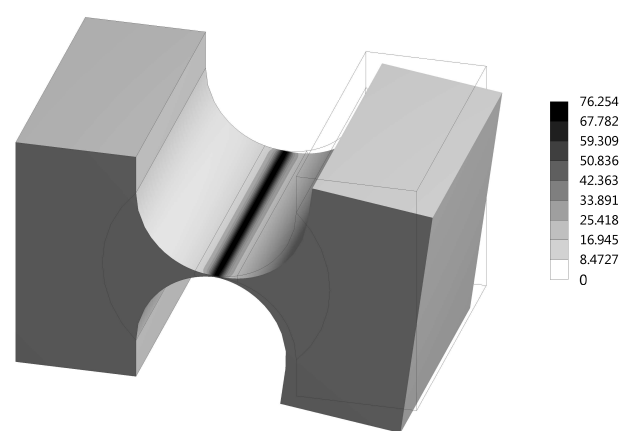

(b)

Figure 3. 3D-FE model of the semi-circular flexure hinge: (a) mesh strategy and boundary conditions; (b) deflected state with bending stress $\left|\sigma_{x}\right|$ in MPa for $\varphi=1^{\circ}$ (scaled by a factor of 21).

Using this meshing strategy and the parameters in Table 2, a sensitivity analysis is conducted with the parameters of the mesh in Appendix A. Results show that the number and transition of elements in the $x$-direction of zone (A) have significant influence on the convergence of the numerical solution of the maximum bending stress $\sigma_{x, \max }$. Other parameters have neglectible influence. Mesh parameter values are then selected, and convergence is studied. The total number of elements amounts to 18,912 to achieve convergence of the numerical solution of the bending stress $\left|\sigma_{x}\right|$ (see Figure $3 b$ ).

The bending stiffness of the hinge is determined using Equation (2). The bending moment $M$ in the model is generated by a shear force $F$ parallel to the $z$-axis applied to the edge of the free end with a lever arm of $L / 2$ to the assumed rotational center. The rotation angle $\varphi$ is calculated from the resulting displacements of two points of the free end (see Figure 2). Using the parameter values in Table 2, the bending stiffness of the semi-circular flexure hinge is calculated according to various models (for the corresponding assumptions refer to Table 1). The results are shown in Table 3. Not every model allows the calculation of large deformations, which requires a nonlinear consideration. In order to achieve a comparability of the results, the rotation angle of nonlinear models is limited to one degree $\left(\varphi=1^{\circ}\right)$. In this deflection range the validity of the linear beam theory can be assumed with sufficient accuracy. Applications which demand the highest precision often operate at small deflections to ensure linear material behavior [28] and to avoid stiffening effects, e.g., anticlastic bending [23]. 
Table 3. Bending stiffness $k_{\varphi}$ according to different models.

\begin{tabular}{lc}
\hline Model & $\begin{array}{c}\boldsymbol{k}_{\boldsymbol{\varphi}} \\
\mathbf{( N m m / r a d )}\end{array}$ \\
\hline Exact equation by Paros and Weisbord [12] & 16.243 \\
Exact equation by Lobontiu [9] & 16.243 \\
Approximate equation by Tseytlin [16] & 18.542 \\
Design tool by Henning et al. [18] & 16.173 \\
Design equation by Linß et al. [17] & 15.887 \\
Linear 3D-FE model & 18.029 \\
Nonlinear 3D-FE model & 18.032 \\
\hline
\end{tabular}

There are significant differences between the results of analytical equations and the 3D-FE model. The result of 3D-FE model is about 10-12\% stiffer than the analytical results, except for the equation according to Tseytlin [16]. A modified elastic modulus $E^{\prime}=E /\left(1-v^{2}\right)$, which applies for plates and thin films, is considered in this equation [29]. The factor $1 /\left(1-v^{2}\right)$ relates the flexural rigidity of a beam assuming a plane strain state and that of a beam assuming a plane stress state. The consideration of this factor corresponds to the model assumption of the plane strain state, which results in a better agreement with the results of the 3D-FE model with the parameters in Table 2. However, the results of the equation by Tseytlin [16] still show deviations not suitable for many other demanding precision applications with different typical geometric ratios. Thus, the development of more accurate equations based on a thorough understanding of the deviation causes becomes necessary.

\section{Comparison between Analytical and Finite Element Models}

To better understand the observed behavior, the relative stiffness deviation of the analytical results compared to the results of the 3D-FE model is shown in Figure 4 for minimum notch heights of 0.05 to $0.1 \mathrm{~mm}$. Other parameter values and the number of elements remain constant. The analytical equation by Lobontiu [9] is no longer considered, since the results are always identical to those by Paros and Weisbord [12]. In addition, two nonlinear 2D-FE models, one assuming a plane stress state and one a plane strain state, are used for comparison. The mesh with 2D-elements is equal to the side face of the 3D-mesh.

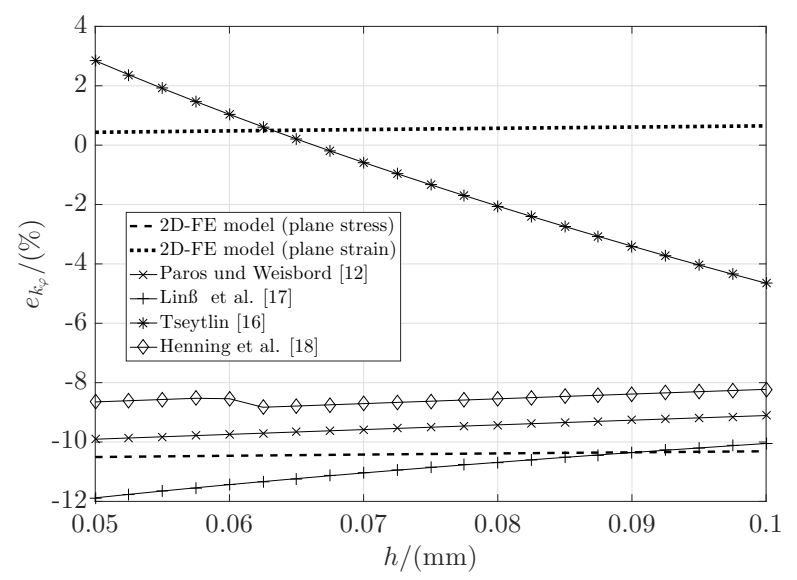

Figure 4. Relative stiffness deviation to the 3D-FE model.

Figure 4 shows that:

- For the parameter values considered, the results of the 3D-FE model are closer to the 2D-FE plane strain model.

- The minimum notch height $h$ or the $h / R$ ratio in this geometry range has a small influence on the deviation to the 3D-FE model, except for the equation by Tseytlin [16]. 
The state of stress of a prismatic body, in which loading is uniformly distributed over its width, generally lies between two limiting cases: the plane stress state (for $b \rightarrow 0$ ) and the plane strain state $($ for $b \rightarrow \infty$ ) [30]. In the plane stress state, the normal stress in the out-of-plane direction is assumed to be zero. In contrast, in a plane strain state, this stress is not zero, but is constantly distributed across the width and its value depends on the normal stresses in the $x-y$ plane. As a result, the bending strain is reduced according to Hooke's law, increasing the bending stiffness by a factor of approximately $1 /\left(1-v^{2}\right)$.

An out-of-plane normal stress is always present in the flexure hinge, which is not constantly distributed across the width and becomes zero at the boundary faces, i.e., anticlastic bending [23]. In Figure 5, the distribution of this stress is represented by means of a dimensionless out-of-plane normal stress $\tilde{\sigma}_{z}$ over the dimensionless $z$-coordinate $\tilde{z}=z / b$ in the outer transverse fiber at the thinnest region of the hinge $(h=0.05 \mathrm{~mm})$. The dimensionless stress $\tilde{\sigma}_{z}$ is formulated as the quotient of the normal stress in one point and the maximum value across the width:

$$
\tilde{\sigma}_{z}=\frac{\sigma_{z}}{\sigma_{z, \max }}
$$

The distribution of the out-of-plane normal stress according to Figure 5 shows that the state of stress of the 3D-FE model changes from plane stress to the plane strain with increasing width. As the width gets larger, the distribution of the out-of-plane normal stress becomes more uniform. Above a certain value of $b$, the behavior converges near to plane strain and the stiffness becomes almost linearly proportional to the width. According to the analytical equations and 2D-FE models, the bending stiffness is always linearly proportional to the width $b$ (see Appendix B). However, the behavior of $\sigma_{z}$ demonstrates a nonlinear relationship, meaning that the deviation depends on the width $b$.

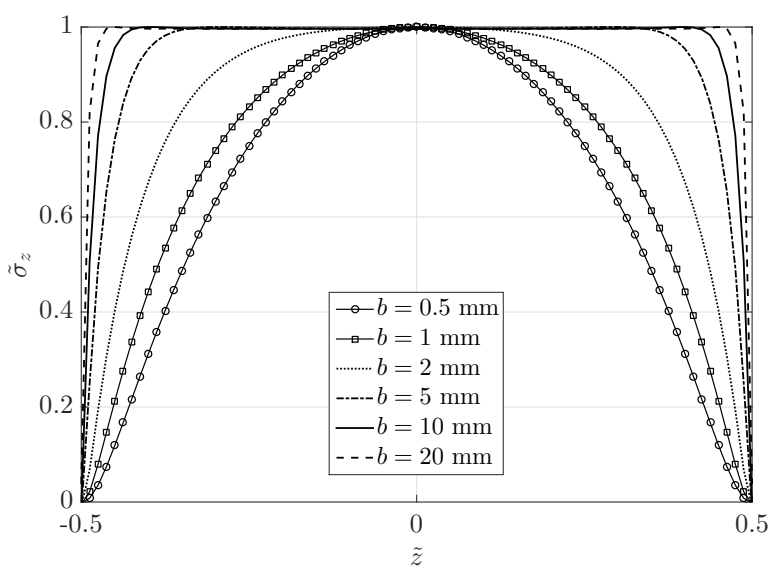

Figure 5. Dimensionless out-of-plane normal stress along the dimensionless $z$-coordinate for different width values $b$ for $h=0.05 \mathrm{~mm}$.

In [21], it was demonstrated that the deviation between analytical equations and 2D-FE models in semi-circular flexure hinges depends only on the $h / R$ ratio. This is related to the stress concentration occurring on the notch contour, which increases the bending strain in 2D-FE models, reducing the stiffness. Then, the deviation between the 3D-FE model and analytical equations must depend on $h$, $R$ and $b$, and possibly on other parameters such as $H$ and $L$. Due to its importance and widespread use, the equation based on Euler-Bernoulli's beam theory by Paros and Weisbord [12] is used for comparison. The equations by Linß et al. [17] and Tseytlin [16] do not apply for the geometries considered here, since they exceed the theoretical limits in Figure 4. This is due to their approximation nature. The equation by Tseytlin [16] delivers results closer to the 3D-FE model using the parameters in Table 2 but can be highly inaccurate for other geometries [21]. 
The relative deviation of FE models to the equation by Paros and Weisbord [12] is calculated for the geometric ratios $h / R$ and $b / h$ and is presented in Figure 6a. Results show the expected multi-criteria effect in the deviation of the 3D-FE model but is also present between 3D- and 2D-FE models, especially for low $b / h$ values. Nevertheless, when fixing $h / R$ to a certain value $(h / R=0.025)$ the deviations becomes constant for discrete $b / h$ ratios (see Figure $6 b$ ). From this, it can be concluded that the deviation of the results of analytical models to the 3D-FE model depends only on the ratios $h / R$ and $b / h$ rather than on absolute values of the geometrical parameters. This is promising for the development of accurate design equations for the calculation of the bending stiffness of flexure hinges with circular contours. It should be noted, however, that the total height $H$ may have a relevant effect for thicker hinges or incomplete circular contours $(l<2 R)$ [17]. The study of a complete semi-circular contour is very convenient because it can be described by only two parameters, $h$ and $R$. Other contours are described by three or more parameters, e.g., elliptical contour, which increases the complexity of the deviation.

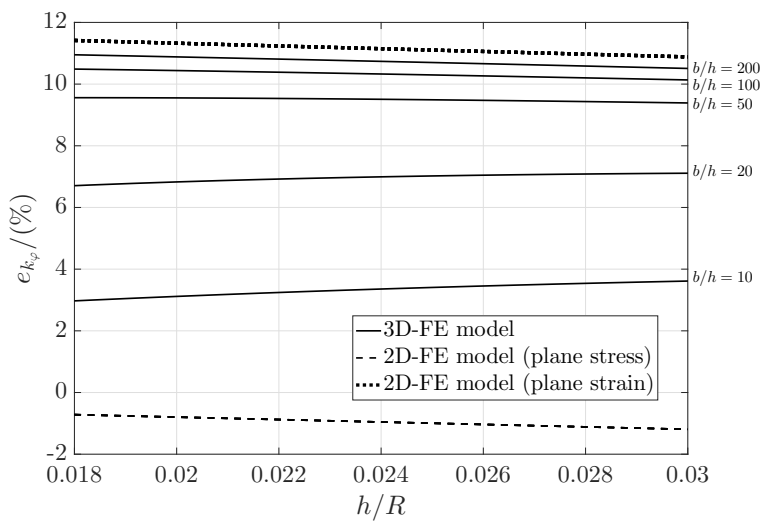

(a)

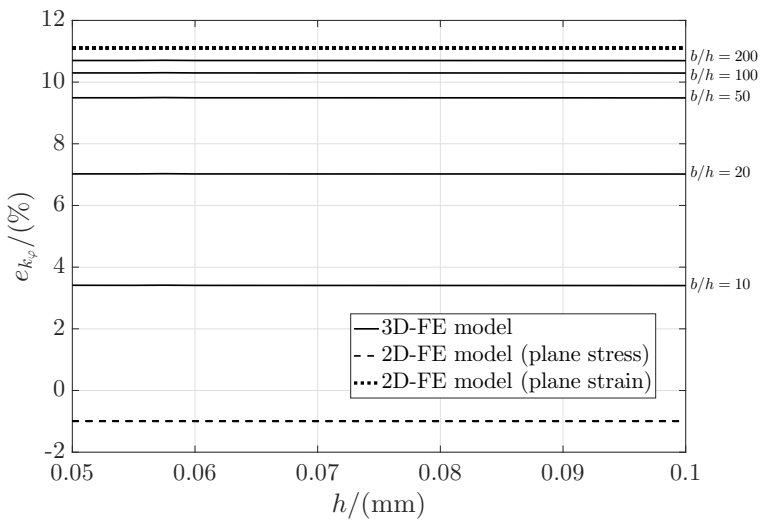

(b)

Figure 6. Relative stiffness deviation of the FE models to the analytical equation by Paros and Weisbord [12] for different $b / h$ ratios and: (a) different $h / R$ ratios $(R=3 \mathrm{~mm}) ;(\mathbf{b}) h / R=0.025$.

In [22], a modified elastic modulus, which depends only on $b / h$, was presented as a correction factor to reduce the deviation when using a 2D-FE model assuming plane stress. However, this factor does not take into account the relationship between $h / R$ and $b / h$ and was derived using a discrete $h / R$ ratio of 0.8 . This causes large deviations for small $b / h$ values (see Figure 7 ). A more precise equation for calculating the bending stiffness based on FEM-based correction factors is derived in the following section.

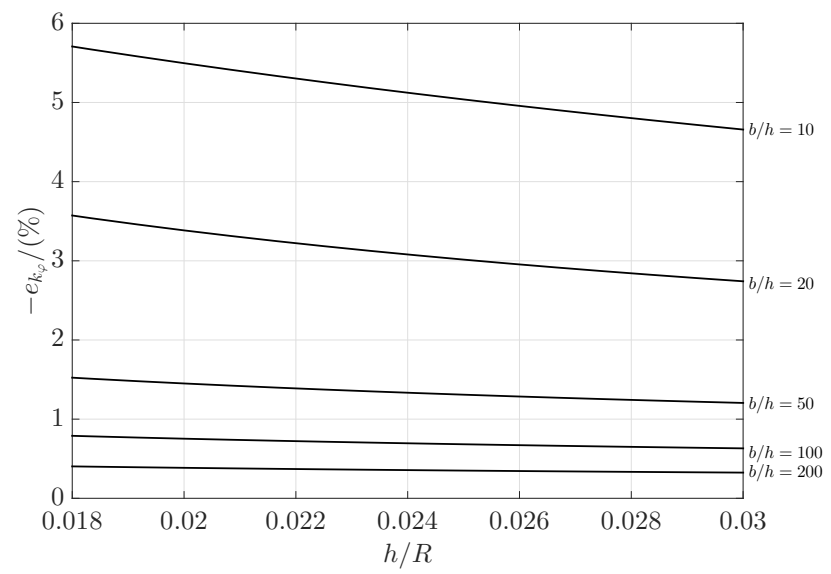

Figure 7. Relative stiffness deviation of the 3D-FE model to the 2D-FE plane stress model considering the correction factor proposed by Zettl et al. [22] for different $h / R$ and $b / h$ ratios. 


\section{Correction Factors for the Accurate Determination of the Bending Stiffness}

The described deviation between model results serves as the basis for the development of a correction factor for the results of analytical equations based on the assumptions of the Euler-Bernoulli beam theory (BT) (see Table 1). The factor equation is approximated by analyzing the different effects separately and is formulated as follows:

$$
\frac{k_{\varphi}^{*}}{k_{\varphi}}=f_{B T}^{3 D}=f_{2 D}^{3 D} f_{B T}^{2 D}
$$

In this case, $f_{B T}^{2 D}$ is a correction factor for the deviation between the 2D-FE plane stress model and the beam-theory equation by Paros and Weisbord [12], which is a result of an inaccurate consideration of the stress distribution in the contour. The factor $f_{2 D}^{3 D}$ corrects the deviation between the 2D-FE plane stress model and the 3D-FE model due to neglecting of the out-of-plane normal stress. Thus, the total correction factor $f_{B T}^{3 D}$, which takes into account the effects of both contour and width, can be calculated.

The correction factors $f_{B T}^{2 D}$ and $f_{2 D}^{3 D}$ can be calculated from the respective relative stiffness deviations $e_{2 D}^{3 D}$ and $e_{B T}^{2 D}$ :

$$
f_{2 D}^{3 D}=e_{2 D}^{3 D}+1, f_{B T}^{2 D}=e_{B T}^{2 D}+1 .
$$

The stiffness deviations $e_{2 D}^{3 D}$ and $e_{B T}^{2 D}$ are approximated as functions of the corresponding parameters $h / R$ and $b / h$ using calculated support points. The results in Figure $6 \mathrm{~b}$ show that the uniform scaling of the hinge does not affect the deviation. Thus, the calculation of the support points can be conducted for a fixed parameter, e.g., the radius $R$, and the resulting function would still apply to any parameter values under the same geometric ratios. The results of the support points are calculated for $h / R$ from 0.015 to 0.15 and $b / h$ from 10 to 100, with more calculation points by $b / h$ between 10 and 60 due to the strong nonlinearity in this region, and a fixed radius $R$ of $3 \mathrm{~mm}$.

Since the relative deviation $e_{B T}^{2 D}$ depends linearly on the $h / R$ ratio, it can be approximated with a polynomial of first order (see Figure 8). This results in the correction factor for the deviation of the analytically determined bending stiffness to the plane stress state (2D-FE model) to:

$$
f_{B T}^{2 D}=1-0.3866 \frac{h}{R}
$$

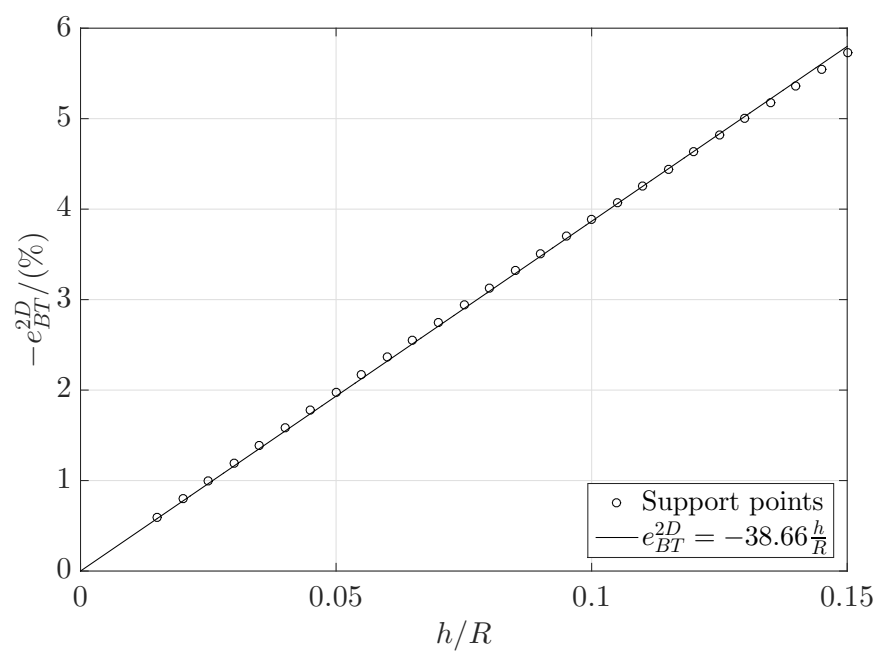

Figure 8. Relative stiffness deviation of the 2D-FE plane stress model to the analytical equation by Paros and Weisbord [12] for different $h / R$ ratios.

The deviation $e_{2 D}^{3 D}$ is influenced by various parameters, which makes the search for a suitable function more complicated. An approximation of $e_{2 D}^{3 D}$ as a function of $h / R$ and $b / h$ would apply only 
to the material used. The effect of the transverse contraction is still to be considered. Conveniently, $e_{2 D}^{3 D}$ should always converge to $v^{2} /\left(1-v^{2}\right)$ (see Figure 9). It is assumed that a normalized deviation

$$
\tilde{e}_{2 D}^{3 D}=\frac{e_{2 D}^{3 D}}{\frac{v^{2}}{1-v^{2}}}
$$

depends only on $h / R$ and $b / h$. In essence, $\tilde{e}_{2 D}^{3 D}$ serves as a factor for the transition from the plane stress state $\left(\tilde{e}_{2 D}^{3 D}=0\right)$ to the plane strain state $\left(\tilde{e}_{2 D}^{3 D}=1\right)$. Based on the similarity of the behavior of the deviation for each discrete $h / R$ value shown in Figure 9, $\tilde{e}_{2 D}^{3 D}$ is adjusted as a function of $b / h$, where the corresponding coefficients are functions of $h / R$. The proposed function has the following form

$$
\tilde{e}_{2 D}^{3 D}=\arctan \left(c \frac{h}{R}\right)+d,
$$

where $c$ and $d$ are the coefficients to be approximated. The coefficients are adjusted according to the following functions:

$$
\begin{gathered}
c=0.653\left(\frac{h}{R}\right)^{0.4239}, \\
d=-0.1029 \frac{h}{R}-0.5565 .
\end{gathered}
$$

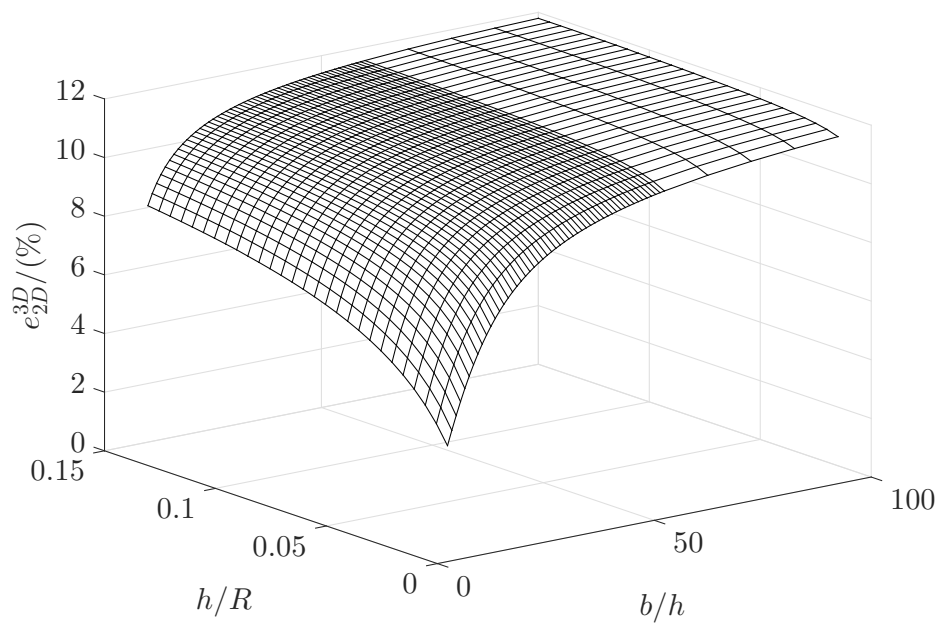

Figure 9. Relative stiffness deviation of the 3D-FE model to the 2D-FE plane stress model for different $h / R$ and $b / h$ ratios.

From this, the correction factor $f_{2 D}^{3 D}$ can be derived:

$$
f_{2 D}^{3 D}=1+\left[\arctan \left(0.653\left(\frac{h}{R}\right)^{0.4239} \frac{b}{h}\right)-0.1029 \frac{h}{R}-0.5565\right]\left(\frac{v^{2}}{1-v^{2}}\right) .
$$

Using the two introduced correction factors $f_{B T}^{2 D}$ in Equation (6) and $f_{2 D}^{3 D}$ in Equation (11), the deviation between the 3D-FE model and the analytical results of the equation of Paros and Weisbord [12] is recalculated (see Figure 10). The use of this approach provides a very good agreement with the results of the 3D-FE model for the presented geometry range shown with a maximum deviation of only $0.23 \%$, which is much lower than that of the existing model equations. Thus, the correction factors can be used to correct other modeling equations based on the same assumptions, too (see Table 1). 


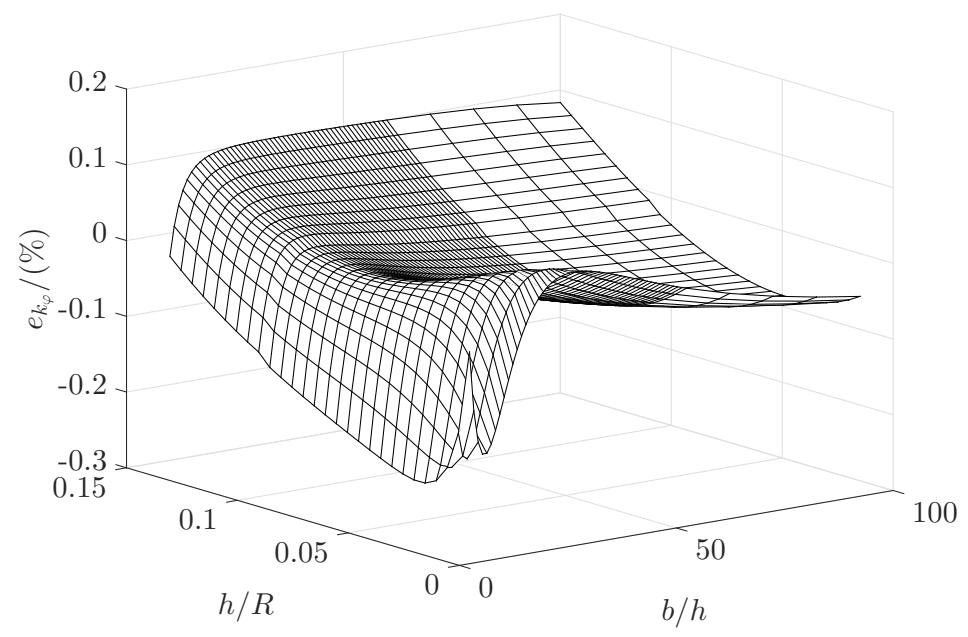

Figure 10. Relative stiffness deviation of the 3D-FE model to the analytical equation by Paros and Weisbord [12] considering the estimated correction factors for different $h / R$ and $b / h$ ratios.

\section{Verification of Results in Application Examples}

To verify the accuracy of the presented approach, the corrected bending stiffness $k_{\varphi}^{*}$ is calculated using parameter values from semi-circular flexure hinges existing in the literature and compared to the bending stiffness $k_{\varphi}^{3 D}$ according to the 3D-FE model of Section 3. Parameter values are presented in Table 4. The values of the total height $H$ and total length $L$ used in the applications are not taken into account for the calculation but are rather selected to model a complete semi-circular contour. Since the values of material properties are not consistent among sources, the elastic constants $E$ and $v$ are extracted from [27]. The bending stiffness calculated using the equation by Paros and Weisbord [12] (noted as $k_{\varphi}$ here) is also used for comparison. The relative stiffness deviation to the 3D-FE model results of the corrected bending stiffness and of the bending stiffness according to Paros and Weisbord [12], $e_{k_{\varphi}^{*}}$ and $e_{k_{\varphi}}$, respectively, are presented in Table 5. Results show the significant reduction in the deviation, especially for the thinnest hinges. Even for values outside the range of application, the corrections factors still represent a very good alternative due to the conditions of their estimation.

Table 4. Considered flexure hinge parameter values in different application examples.

\begin{tabular}{cccccclll}
\hline Nr. & $\begin{array}{c}\boldsymbol{h} \\
(\mathbf{m m})\end{array}$ & $\begin{array}{c}\boldsymbol{R} \\
(\mathbf{m m})\end{array}$ & $\begin{array}{c}\boldsymbol{b} \\
(\mathbf{m m})\end{array}$ & $\begin{array}{c}\boldsymbol{E} \\
(\mathbf{G P a})\end{array}$ & $\boldsymbol{v}$ & Material & Application & Ref. \\
\hline 1 & 0.04 & 4.98 & 15 & 207 & 0.30 & Steel alloy & Investigation on single hinge & {$[6,7]$} \\
2 & 0.05 & 8 & 10 & 128 & 0.30 & Beryllium-copper & Watt balance & {$[31]$} \\
3 & 0.07 & 5 & 25 & 114 & 0.34 & Titanium alloy & Parallel manipulator & {$[32]$} \\
4 & 0.25 & 1.5 & 20 & 141 & 0.29 & Invar & Positioning stage & {$[4]$} \\
5 & 0.25 & 5 & 15 & 71 & 0.33 & Aluminum alloy & Amplifier mechanism & {$[33]$} \\
6 & 0.30 & 5 & 6 & 71 & 0.33 & Aluminum alloy & Investigation on single hinge & {$[17]$} \\
7 & 0.40 & 15 & 15 & 114 & 0.34 & Titanium alloy & Parallel manipulator & {$[34]$} \\
8 & 0.50 & 3 & 15 & 71 & 0.33 & Aluminum alloy & Amplifier mechanism & {$[33]$} \\
9 & 0.60 & 12 & 12 & 207 & 0.30 & Steel alloy & Positioning stage & {$[35]$} \\
\hline
\end{tabular}


Table 5. Calculated flexure hinge bending stiffness results.

\begin{tabular}{cccccccccc}
\hline Nr. & $\boldsymbol{h} / \boldsymbol{R}$ & $\boldsymbol{b} / \boldsymbol{h}$ & $f_{\boldsymbol{B} \boldsymbol{T}}^{2 D}$ & $f_{\mathbf{2} \boldsymbol{D}}^{3 D}$ & $\begin{array}{c}\boldsymbol{k}_{\boldsymbol{\varphi}} \\
\mathbf{( N m m} / \mathbf{r a d})\end{array}$ & $\begin{array}{c}\boldsymbol{k}_{\boldsymbol{\varphi}}^{*} \\
\mathbf{( N m m} / \mathbf{r a d})\end{array}$ & $\begin{array}{c}\boldsymbol{k}_{\boldsymbol{\varphi}}^{3 D} \\
\mathbf{( N m m} / \mathbf{r a d})\end{array}$ & $\begin{array}{c}\boldsymbol{e}_{\boldsymbol{k}_{\varphi}} \\
\mathbf{( \% )}\end{array}$ & $\begin{array}{c}\boldsymbol{e}_{\boldsymbol{k}_{\boldsymbol{\varphi}}^{*}} \\
\mathbf{( \% )}\end{array}$ \\
\hline 1 & 0.008 & 375 & 0.997 & 1.097 & 31.53 & 34.48 & 34.45 & -8.486 & 0.090 \\
2 & 0.006 & 200 & 0.998 & 1.094 & 17.91 & 19.54 & 19.53 & -8.292 & 0.063 \\
3 & 0.014 & 357 & 0.995 & 1.129 & 117.09 & 131.47 & 131.30 & -10.829 & 0.127 \\
4 & 0.167 & 80 & 0.936 & 1.088 & 5200.76 & 5292.89 & 5300.39 & -1.880 & -0.141 \\
5 & 0.050 & 60 & 0.981 & 1.112 & 1059.51 & 1155.67 & 1155.03 & -8.270 & 0.055 \\
6 & 0.060 & 20 & 0.977 & 1.093 & 669.39 & 714.66 & 714.72 & -6.343 & -0.008 \\
7 & 0.133 & 37.5 & 0.948 & 1.118 & 7189.32 & 7625.27 & 7629.22 & -5.766 & -0.052 \\
8 & 0.167 & 30 & 0.936 & 1.109 & 7856.47 & 8148.34 & 8161.33 & -3.735 & -0.159 \\
9 & 0.171 & 20 & 0.934 & 1.083 & $26,778.32$ & $27,071.77$ & $27,124.71$ & -1.277 & -0.195 \\
\hline
\end{tabular}

\section{Conclusions}

In high-precision flexure hinge-based measurement instruments an accurate model of the deformation behavior is required. In this paper, the existing deviation between different FE models and analytical equations available in the literature for calculating the bending stiffness of thin semi-circular flexure hinges is explained. Due to the large aspect ratios of thin hinges, in particular the ratio of width $b$ to minimum notch height $h$, an inaccurate modeling of the flexure hinge exists due to the assumption of a plane stress state. Known analytical equations are based almost exclusively on this assumption. The resulting relative stiffness deviations of up to $12 \%$ can be explained by the neglection of the existing out-of-plane normal stress and an associated underestimation of the bending stiffness. It is demonstrated that the deviation of the analytical equations based on the Euler-Bernoulli BT to the 3D-FE model depends on two geometric ratios: width to minimum notch height $b / h$ and minimum notch height to radius $h / R$. In order to avoid complex FE analyses, two correction factors for the analytical equations based on Euler-Bernoulli's BT are proposed for typical flexure hinges with a semi-circular contour: one correction factor for the deviation of BT to the plane stress state and one for the deviation between the plane stress state and the 3D-FE model caused by the out-of-plane stress. These correction factors depend on two dimensionless parameters that describe the geometry of the flexure hinge: $h / R$ and $b / h$. Using both correction factors, the deviation from the results of the 3D-FE model for the considered wide range of geometric parameters becomes smaller than $0.3 \%$. Thus, with the proposed correction factors, designers of compliant mechanisms have a simple possibility at hand that allows an accurate calculation of the bending stiffness for very different geometries of semi-circular flexure hinges. The results obtained this way show a good correlation with elaborated 3D-FE models.

Author Contributions: Conceptualization, M.A.T.M., M.D. and S.L.; methodology, M.A.T.M. and M.D.; formal analysis, M.A.T.M. and M.D.; writing—original draft preparation, M.A.T.M.; writing—review and editing, M.D., S.L., L.Z., T.F. and R.T.; supervision, M.D. and R.T.; project administration, M.D. and R.T.; funding acquisition, L.Z. and R.T.

Funding: The presented content is closely related to several funded projects at TU Ilmenau. The authors would like to thank the German Research Foundation (DFG) for the financial support of the Research Training Group "Tip- and laser-based 3D-Nanofabrication in extended macroscopic working areas" (GRK 2182) and the projects with the Grant Nos.: TH845/7-1 and TH845/5-2.

Conflicts of Interest: The authors declare no conflict of interest. The funding sponsors had no role in the analyses or interpretation of results within the study; in the writing of the manuscript, and in the decision to publish the results. 


\section{Abbreviations}

The following abbreviations are used in this manuscript:

FE Finite element

3D Three-dimensional

2D Two-dimensional

BT Beam theory

\section{Appendix A}

Mesh sensitivity analysis:

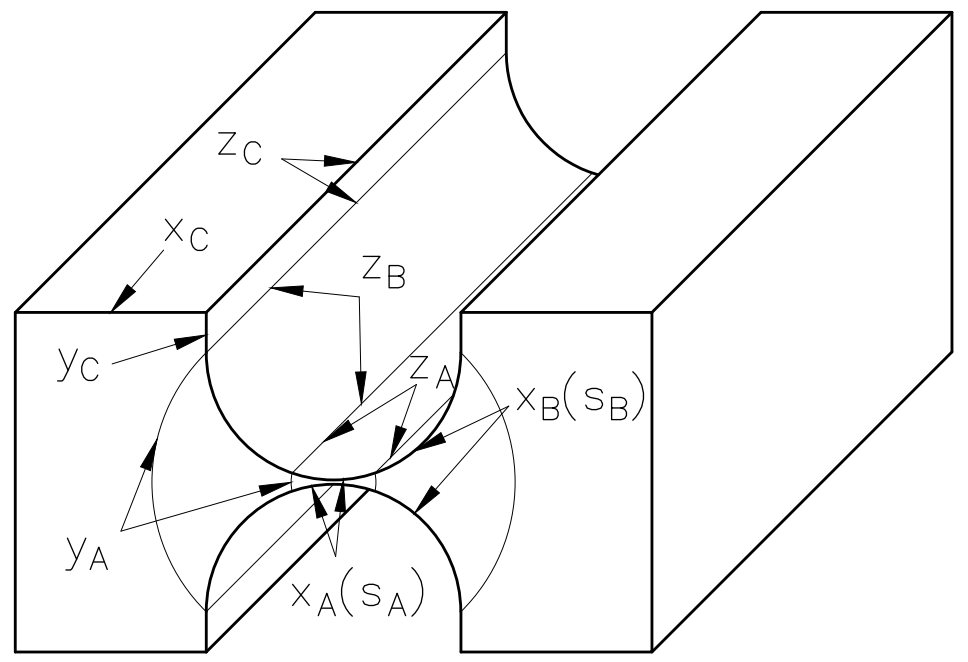

Figure A1. Local refinements.

Table A1. Mesh parameters.

\begin{tabular}{|c|c|c|}
\hline Parameter & Description & Value \\
\hline$x_{A}$ & Elements along the length of zone A & 10-100 \\
\hline$x_{B}$ & Elements along the length of zone B & $3-30$ \\
\hline$x_{C}$ & Elements along the length of zone $\mathrm{C}$ & $1-10$ \\
\hline$y_{A}$ & Elements along the height of zones A and B & $2-10$ \\
\hline$y_{C}$ & Additional elements along the height of zone $\mathrm{C}$ & $1-5$ \\
\hline$s_{A}$ & $\begin{array}{l}\text { Transition of elements along the length of zone A towards } \\
\text { the center of the hinge }\end{array}$ & $0-1$ \\
\hline$s_{A}$ & $\begin{array}{l}\text { Transition of elements along the length of zone B towards } \\
\text { the center of the hinge }\end{array}$ & $0-1$ \\
\hline$A R$ & Limiting aspect ratio & $5-10$ \\
\hline$z_{A}$ & Elements along the width of zone A & $\frac{b y_{A}}{h A R}$ \\
\hline$z_{B}$ & Elements along the width of zone B & $b y_{A}$ \\
\hline$z_{C}$ & Elements along the width of zone $\mathrm{C}$ & $\begin{array}{c}\overline{\sqrt{10} h A R} \\
\frac{b y_{A}}{(h+2 R)}\end{array}$ \\
\hline
\end{tabular}


Table A2. Total effect sensitivity indices.

\begin{tabular}{ccc}
\hline Parameter & $\mathbf{S}_{\sigma_{x}, \max }$ & $\mathbf{S}_{\text {nodes }}$ \\
\hline Model & 1.000 & 1.000 \\
$x_{A}$ & 0.847 & 0.325 \\
$x_{B}$ & 0.075 & 0.081 \\
$x_{C}$ & 0.000 & 0.004 \\
$y_{A}$ & 0.048 & 0.669 \\
$y_{C}$ & 0.000 & 0.003 \\
$s_{A}$ & 0.249 & 0.003 \\
$s_{C}$ & 0.000 & 0.002 \\
$A R$ & 0.064 & 0.115 \\
\hline
\end{tabular}

\section{Appendix B}

Paros and Weisbord (1965), full equation [12]:

$$
\begin{aligned}
k_{\varphi}= & \frac{2 E b R^{2}}{3}\left(2 \xi+\xi^{2}\right)\left\{\left[\frac{1+\xi}{\zeta^{2}}+\frac{3+2 \xi+\xi^{2}}{\zeta\left(2 \xi+\xi^{2}\right)}\right] \sqrt{1-(1+\xi-\zeta)^{2}}+\right. \\
& {\left.\left[\frac{6(1+\xi)}{\left(2 \xi+\xi^{2}\right)^{3 / 2}}\right] \tan ^{-1}\left(\sqrt{\frac{2+\xi}{\xi}} \frac{(\zeta-\xi)}{\sqrt{1-(1+\xi-\zeta)^{2}}}\right)\right\}^{-1} }
\end{aligned}
$$

with $\xi=h / 2 R$ and $\zeta=1+\xi$.

Tseytlin (2002) [16]:

For thin hinges, $h / R \leq 0.07$ :

$$
k_{\varphi}=\frac{E b h^{2}}{16}\left\{1+\left[1+0.1986\left(\frac{2 R}{h}\right)\right]^{1 / 2}\right\}^{-1} .
$$

Lobontiu (2003) [9]:

$$
\begin{gathered}
k_{\varphi}=\frac{E b h^{3}(2 R+h)(4 R+h)^{3}}{24 R}\left[h(4 R+h)\left(6 R^{2}+4 R h+h^{2}\right)+\right. \\
\left.6 R(2 R+h)^{2} \sqrt{h(4 R+h)} \tan ^{-1}\left(\sqrt{1+\frac{4 R}{h}}\right)\right]^{-1} .
\end{gathered}
$$

Linß et al. (2017) [17]:

$$
k_{\varphi}=k_{M 1} E b\left(\frac{l}{H}\right)^{-k_{M 2}}\left(\frac{h}{H}\right)^{2+k_{M 2}} H^{2}
$$

with $k_{M 1}=107.9 \times 10^{-3}$ and $k_{M 2}=0.52$ for circular contours.

\section{References}

1. Darnieder, M.; Pabst, M.; Wenig, R.; Zentner, L.; Theska, R.; Fröhlich, T. Static behavior of weighing cells. J. Sens. Sens. Syst. 2018, 7, 587-600. [CrossRef]

2. Smith, S.T.; Chetwynd, D.G.; Bowen, D.K. Design and assessment of monolithic high precision translation mechanisms. J. Phys. E Sci. Instrum. 1987, 20, 977-983. [CrossRef]

3. Quinn, T.J.; Speake, C.C.; Davis, R.S. A 1 kg Mass Comparator Using Flexure-Strip Suspensions: Preliminary Results. Metrologia 1986, 23, 87-100. [CrossRef] 
4. Scire, F.E.; Teague, E.C. Piezodriven 50-micrometer range stage with subnanometer resolution. Rev. Sci. Instrum. 1978, 49, 1735. [CrossRef] [PubMed]

5. International Federation for the Promotion of Mechanism and Machine Science. IFToMM Dictionaries, 2014. Available online: http://www.iftomm-terminology.antonkb.nl/2057_1031/frames.html (accessed on 14 October 2018).

6. Hensgen, L. Auswirkung der Drahtfunkenerosion auf die Mechanischen Eigenschaften von Festkörpergelenken. Ph.D. Thesis, RWTH Aachen, Aachen, Germany, 2017.

7. Schönen, D.; Lersch, S.; Hüsing, M.; Corves, B.; Klocke, F.; Hensgen, L.; Klink, A. Entwicklung, Konstruktion und Anwendung Eines Prüfstands zur Ermittlung der Ertragbaren Lastzyklen Filigraner Hochgenauer Stoffschlüssiger Gelenke; 11. Kolloquium Getriebetechnik: Garching, Germany, 28-30 September 2015; pp. 169-181.

8. Linß, S. Ein Beitrag zur Geometrischen Gestaltung und Optimierung Prismatischer Festkörpergelenke in Nachgiebigen Koppelmechanismen. Ph.D. Thesis, Technische Universität Ilmenau, Ilmenau, Germany, 2015.

9. Lobontiu, N. Compliant Mechanisms: Design of Flexure Hinges; CRC Press: Boca Raton, FL, USA, 2003.

10. Zentner, L. Nachgiebige Mechanismen; De Gruyter Oldenbourg: Berlin, Germany, 2014; ISBN 978-3-486-85890-7.

11. Howell, L.L.; Midha, A. A Method for the Design of Compliant Mechanisms with Small-Length Flexural Pivots. J. Mech. Des. 1994, 116, 280-290. [CrossRef]

12. Paros, J.; Weisbord, L. How to design flexure hinges. Mach. Des. 1965, 25, 151-156.

13. Li, Q.; Pan, C.; Xu, X. Closed-form compliance equations for power-function-shaped flexure hinge based on unit-load method. Precis. Eng. 2013, 37, 135-145. [CrossRef]

14. Zhu, Z.; Zhou, X.; Wang, R.; Liu, Q. A simple compliance modeling method for flexure hinges. Sci. China Technol. Sci. 2015, 58, 56-63. [CrossRef]

15. Dirksen, F.; Lammering, R. On mechanical properties of planar flexure hinges of compliant mechanisms. Mech. Sci. 2011, 2, 109-117. [CrossRef]

16. Tseytlin, Y.M. Notch flexure hinges: An effective theory. Rev. Sci. Instrum. 2002, 73, 3363-3368. [CrossRef]

17. Linß, S.; Schorr, P.; Zentner, L. General design equations for the rotational stiffness, maximal angular deflection and rotational precision of various notch flexure hinges. Mech. Sci. 2017, 8, 29-49. [CrossRef]

18. Henning, S.; Linß, S.; Zentner, L. detasFLEX-A computational design tool for the analysis of various notch flexure hinges based on non-linear modeling. Mech. Sci. 2018, 9, 389-404. [CrossRef]

19. Koster, M.; Rosielle, P.; Reker, E. Constructies voor het nauwkeurig bewegen en positioneren (6). Mikroniek 1992, 32, 68-72.

20. Schotborgh, W.O.; Kokkeler, F.G.; Tragter, H.; van Houten, F.J. Dimensionless design graphs for flexure elements and a comparison between three flexure elements. Precis. Eng. 2005, 29, 41-47. [CrossRef]

21. Yong, Y.K.; Lu, T.F.; Handley, D.C. Review of circular flexure hinge design equations and derivation of empirical formulations. Precis. Eng. 2008, 32, 63-70. [CrossRef]

22. Zettl, B.; Szyszkowski, W.; Zhang, W.J. On Systematic Errors of Two-Dimensional Finite Element Modeling of Right Circular Planar Flexure Hinges. J. Mech. Des. 2005, 127, 782-787. [CrossRef]

23. Campanile, L.; Jähne, R.; Hasse, A. Exact analysis of the bending of wide beams by a modified elastica approach. J. Mech. Eng. Sci. 2011, 225, 2759-2764. [CrossRef]

24. Eastman, F.S. Flexure Pivots to Replace Knife Edges and Ball Bearings: An Adaptation of Beam-Column Analysis; University of Washington: Seattle, WA, USA, 1935.

25. Ryu, J.W.; Gweon, D.G. Error analysis of a flexure hinge mechanism induced by machining imperfection. Precis. Eng. 1997, 21, 83-89. [CrossRef]

26. Bacher, J.P.; Joseph, C.; Clavel, R. Flexures for high precision robotics. Ind. Robot 2002, 29, 349-353. [CrossRef]

27. Callister, W.D. Materials Science and Engineering: An Introduction, 7th ed.; John Wiley \& Sons: New York, NY, USA, 2007.

28. Sydenham, P.H. Elastic design of fine mechanism in instruments. J. Phys. E Sci. Instrum. 1984, 17, 922-930. [CrossRef]

29. Tseytlin, Y. Tractable model for concave flexure hinges. Rev. Sci. Instrum. 2011, 82, 015106. [CrossRef] [PubMed]

30. Timoshenko, S.; Goodier, J. Theory of Elasticity, 2nd ed.; McGraw-Hill Book Company: New York, NY, USA, 1951.

31. Pinot, P.; Genevès, G.; Filtz, J.R.; Larquier, B.; Claudel, P.; Favreau, J.O. Characterization of flexure hinges for the French watt balance experiment. EPJ Web Conf. 2014, 77, 00005. [CrossRef] 
32. Niaritsiry, T.F.; Fazenda, N.; Clavel, R. Simulation analysis of the sources of inaccuracy of a parallel manipulator. In Proceedings of the IEEE International Conference on Robotics, Intelligent Systems and Signal Processing, Changsha, China, 8-13 October 2003; pp. 266-271. [CrossRef]

33. Friedrich, R.; Lammering, R.; Rösner, M. On the modeling of flexure hinge mechanisms with finite beam elements of variable cross section. Precis. Eng. 2014, 38, 915-920. [CrossRef]

34. Ivanov, I.; Corves, B. Stiffness-Oriented Design of a Flexure Hinge-Based Parallel Manipulator. Mech. Based Des. Struc. 2014, 42, 326-342. [CrossRef]

35. Hou, W. Analysis of three nodes flexure hinge element in ultra-precision positioning stage. In Proceedings of the 7th International Conference on Biomedical Engineering and Informatics, BMEI 2014, Dalian, China, 14-16 October 2014; pp. 527-533. [CrossRef]

(C) 2018 by the authors. Licensee MDPI, Basel, Switzerland. This article is an open access article distributed under the terms and conditions of the Creative Commons Attribution (CC BY) license (http:// creativecommons.org/licenses/by/4.0/). 